\title{
Entropy
}

ISSN 1099-4300

www.mdpi.org/entropy/

\section{Behavior of the Thermodynamic Properties of Binary Mixtures near the Critical Azeotrope}

\section{Azzedine Abbaci}

Faculté des Sciences, Département de Chimie, Université Badji Mokhtar, B.P. 12, El-Hadjar, Annaba (23200), Algeria, Tel. +213-38-87-67-65, Fax +213-38-87-67-65, Email: Abbaci_a2002@Yahoo.fr

Received: 26 September 2003 / Accepted: 19 December 2003 / Published: 22 December 2003

\begin{abstract}
In this work we investigate the critical line of binary azeotropic mixtures of acetone-n-pentane. We pinpoint the abnormal behavior of the critical density line as a function of the mole fraction of one of the component and show its influence on other thermodynamic properties such as the volume, the enthalpy and the entropy.
\end{abstract}

Keywords: Azeotropic mixtures, critical line, mole fraction, equation of state.

\section{Introduction}

The liquid-vapor thermodynamic surface of binary mixtures with azeotropes shows three singularities, besides a special point. Two of these singular points are similar to those of ordinary binary mixtures, they represent the critical points of the two pure fluids constituting the binary mixture and they are located at the two extremities of the critical curve, or the critical locus [1]. The third point represents the intercept of the azeotropic line with the critical curve [2]. Finally, the special point is the extremum in the critical temperature; it is a minimum for a positive azeotropy, and a maximum for a negative azeotropy [3]. 
In order to apply the critical point universality class to mixtures, it is important to distinguish between field variables and densities [4]. For one component fluid, the field variables are the pressure $P$, the temperature $T$, and the chemical potential $\mu$. For binary mixtures the field variables are the pressure $P$, the temperature $T$ and the chemical potentials $\mu_{i}$, of the two components, with $i=1$ and $i=2$. These field variables are related by the Gibbs-Duhem equation, furthermore, it is useful to consider the difference between the two chemical potentials [4] such as:

$\Delta=\mu_{1}-\mu_{2}$

Griffiths and Wheeler [4] have pointed out that the critical behavior of binary mixtures is similar to that of one component fluid provided that the field variable $\Delta$ is kept constant. Therefore, the principle of critical point universality class implies that binary mixtures at constant $\Delta$ should satisfy the same universal scaling laws as one component fluid. However, complications arise as a result of the fact that actual experiments for mixtures are carried out at constant mole fraction $x$ and not at constant $\Delta$. Therefore, the variable $\Delta$ is an inconvenient variable, for it diverges in the pure fluid limits as indicated by Leung and Griffiths [5]. Following the earlier work of these latter [5], we therefore, introduce a new field variable $\zeta$ which is in fact a function of $\mu_{1,} \mu_{2}$ and $T$ such that:

$0 \leq \varsigma \leq 1$

where $\varsigma=0$ corresponds to the limit of the pure fluid 1 and $\varsigma=1$ corresponds to the limit of pure fluid 2 . There are a variety of ways to define $\varsigma$ so as to satisfy $(2)[5,6]$. If one holds the mole fraction $x$ constant, then the coexistence curves of binary mixtures have a different topology from that of a onecomponent fluid. On the other hand, when $\varsigma$ is taken to be constant, then the coexistence curves of these binary mixtures should have the same topology as that of a one-component fluid.

In this work we attempt to provide a representation of the critical locus of binary azeotropic mixtures. Specifically, the study of the critical parameters as functions of the concentration will play a central role in any accurate representation of mixture data in the critical region. The motivations behind this work are many. For instance, in an earlier work [2] there was a need to formulate a thermodynamic potential for ethane and carbon dioxide mixture. Furthermore, on a different ground, we would like to know to what extend the behavior of the critical parameters may be extended to several thermodynamic properties such as the volume, enthalpy, and the entropy. 
It is therefore of considerable interest to apply the theory to binary mixtures. For the theoretical interpretation of thermodynamic behavior of these mixtures we need to assess their critical parameters as a function of the concentration.

\section{Thermodynamic properties of binary mixtures}

The analogy between pure fluids and fluid mixtures is being established according to the previous section, we then investigate the behavior of some thermodynamic properties such as the volume $V$, the internal energy $U$, the enthalpy $H$, and the entropy $S$.

The derivative of $V=V(P, T, x)$ with respect to the mole fraction $x$ can be written as:

$$
\left(\frac{\partial V}{\partial x}\right)_{c, a z}=\left(\frac{\partial V}{\partial T}\right)_{P, x}\left(\frac{\partial T}{\partial x}\right)_{c, a z}+\left(\frac{\partial V}{\partial P}\right)_{T, x}\left(\frac{\partial P}{\partial x}\right)_{c, a z}+\left(\frac{\partial V}{\partial x}\right)_{P, T}
$$

the last term of this equation can be rewritten as [6]:

$$
\left(\frac{\partial V}{\partial x}\right)_{P, T}=V K_{T, x}\left(\frac{\partial P}{\partial x}\right)_{V, T}
$$

The subscript $(c, a z)$ stands for the critical azeotropy. $K_{T, x}$ is the compressibility of the binary mixtures. For the purpose of implementing our idea, we demand that $x=\zeta$ along the critical line [2]. Consequently, $K_{T, \zeta}$ behaves in the same way as the compressibility of a pure fluid, which we denote by:

$$
K_{T, \varsigma}=\frac{1}{V}\left(\frac{\partial V}{\partial P}\right)_{T}
$$

this quantity is infinite along the critical line. Therefore, when Eq. (4) is substituted into Eq. (3), this latter reduces to the following relation:

$$
\left(\frac{\partial V}{\partial x}\right)_{c, a z}=\left(\frac{\partial V}{\partial T}\right)_{P, x}\left(\frac{\partial T}{\partial x}\right)_{c, a z}+\left(\frac{\partial V}{\partial P}\right)_{T, x}\left(\frac{\partial P}{\partial x}\right)_{c, a z}+V K_{T, \varsigma}\left(\frac{\partial P}{\partial x}\right)_{T}
$$


As mentioned above, the critical temperature of an azeotropic mixture as a function of $x$ has a minimum or a maximum depending on the nature of the mixture. Hence, the first and the second terms of Eq. (6) are identically equal to zero. Moreover, in the last term of Eq. (6), the quantity $K_{T, \zeta} \rightarrow \infty$ on the critical azeotrope of the binary mixtures, this result follows from the basic notion that, at the critical point of pure fluids, the compressibility diverges strongly [7]. Therefore, it comes out that the $V$ - $x$ critical curve possesses an inflection point approaching a vertical tangent, as will be shown in the next section in figure 1. A similar observation is readily seen if one looks at the enthalpy and entropy functions of the binary mixtures. We can take the enthalpy as $H$, or the entropy $S$, along the critical locus. From the basic thermodynamic relation, we have:

$H=U+P V$

where, $U$ is the internal energy of the mixture, $P$ the pressure, and $V$ the total volume. The derivative of the enthalpy with respect to $x$ is given by the following relation:

$$
\left(\frac{\partial H}{\partial x}\right)_{P, T}=\left(\frac{\partial U}{\partial x}\right)_{P, T}+P\left(\frac{\partial V}{\partial x}\right)_{P, T} .
$$

In the absence of any external force, we obtain from the fundamental thermodynamic equation of the total energy $U$ of a closed system, the following relation:

$\left(\frac{\partial U}{\partial V}\right)_{T, x}=T\left(\frac{\partial S}{\partial V}\right)_{T, x}-P$

Furthermore, the quantity $\left(\frac{\partial S}{\partial V}\right)_{T, x}$ can be obtained from the Maxwell relations as:

$\left(\frac{\partial S}{\partial V}\right)_{T, x}=\left(\frac{\partial P}{\partial T}\right)_{V, x}$

By substitution of Eq. (10) into equation (8), we obtain:

$\left(\frac{\partial H}{\partial x}\right)_{P, T}=\left(\frac{\partial U}{\partial x}\right)_{V, T}+T\left(\frac{\partial V}{\partial x}\right)_{P, T}\left(\frac{\partial P}{\partial T}\right)_{V, x}$. 
The same procedure may be done for the behavior of the entropy of the binary mixtures as a function of $x 4$ :

$$
\left(\frac{\partial S}{\partial x}\right)_{P, T}=\left(\frac{\partial S}{\partial x}\right)_{V, T}+\left(\frac{\partial V}{\partial x}\right)_{P, T}\left(\frac{\partial P}{\partial T}\right)_{V, x}
$$

According to theoretical findings [7] and the experimental data of Khazanova and Sominskaya [8-10]; all the following derivatives $\left(\frac{\partial U}{\partial x}\right)_{V, T},\left(\frac{\partial S}{\partial x}\right)_{V, T}$ and $\left(\frac{\partial P}{\partial T}\right)_{V, x}$ are finite. This leads to the conclusion that the isothermals isobars $H-x$ and $S$ - $x$ have the same behavior as that of the isothermals isobars $V-x$ at the critical azeotrope. Making use of the fact that at the critical azeotrope, we have $x=\varsigma$ and using thermodynamic identity at the critical azeotrope, relation (11) reduces to:

$$
\left(\frac{\partial H}{\partial x}\right)_{P, T, a z} \rightarrow \infty \text { and }\left(\frac{\partial^{2} H}{\partial x^{2}}\right)_{P, T, a z} \rightarrow \infty
$$

A similar conclusion can be reached for the entropy, we therefore can write:

$$
\left(\frac{\partial S}{\partial x}\right)_{P, T, a z} \rightarrow \infty
$$

The singular behavior of the thermodynamic properties mentioned above is directly related to the behavior of the critical locus of the studied mixtures. For this purpose we have made an assessment of the critical line.

\section{Investigation of the critical line}

A detailed investigation of the critical parameters of an azeotropic mixture was done by Abbaci et al. [11]. For this purpose we made a choice on an azeotropic mixture such as carbon dioxide-ethane. In this work we take another system that behaves in a similar way as carbon dioxideethane, this system consists of acetone-n-pentane with experimental data measured by Raja et al. [12]. This system presents a positive azeotrope, where the critical locus intercepts the azeotropic locus a point that we call the azeotropic critical point. To correlate the critical density as a function of the 
mole fraction, we fitted the experimental data obtained by Raja et al. [12] to the following relation [11]:

$$
\rho_{c}(x)=\rho_{c}{ }^{(1)}(1-\mathrm{x})+\rho_{c}{ }^{(2)} x+\left(\rho_{1} x+\rho_{2} x^{2}\right)(1-x)
$$

We also fitted the critical temperature of acetone-n-pentane as a function of the mole fraction of n-pentane according to the following equation:

$$
T_{c}(x)=T_{c}^{(1)}(1-\mathrm{x})+T_{c}^{(2)} x+\left(T_{1} x+T_{2} x^{2}+T_{3} x^{3}+T_{4} x^{4}\right)(1-x)
$$

Where $x$ denotes the mole fraction of n-pentane. The first two coefficients in Eqs. (15) and (16) represent the critical densities and the critical temperatures of pure of acetone and n-pentane, whereas, the coefficients $\rho_{j}, T_{j}$ are to be determined by fitting Eq. (15) and Eq. (16) to experimental data obtained by Raja et al. [12]. The values obtained for the coefficients in Eq. (15) and Eq. (16) are presented in Table 1, together with the critical densities as well as the critical temperatures of the pure fluids, namely, acetone and n-pentane.

A plot of the critical temperature as a function of $x$ is presented in figure 1. Plot of the critical density as a function of $x$ is also presented in figure 2. We note on this figure the cusp-like behavior of $\rho(x)$ at the $x=0.63$ that is similar to an inflection point. This point in the critical density does not coincide with the critical temperature minimum as reported by Raja et al. [12]. This fact is in contradiction with what happens in the case of ethane-carbon dioxide mixture, where the critical temperature minimum coincides with the discontinuity in the critical density [11]. It is clear from figure 2 , that we were unsuccessful to reproduce the inflection point in the critical density using simple polynomials.

Table 1: Critical-line parameters for acetone-n-pentane

\begin{tabular}{|c|}
\hline Equation (16) for $\rho_{c}\left(\mathrm{~m}^{3} / \mathrm{mol}\right): \rho_{c}{ }^{(1)}=311.0, \rho_{c}{ }^{(2)}=213.0, \rho_{1}=38.14, \rho_{2}=-15.60$ \\
\hline Equation (17) for $T_{\mathrm{c}}(\mathrm{K}): T_{\mathrm{c}}{ }^{(1)}=469.80, T_{\mathrm{c}}^{(2)}=507.60, T_{1}=-69.309, T_{2}=87.175$, \\
$T_{3}=-204.59, T_{4}=128.68$
\end{tabular}




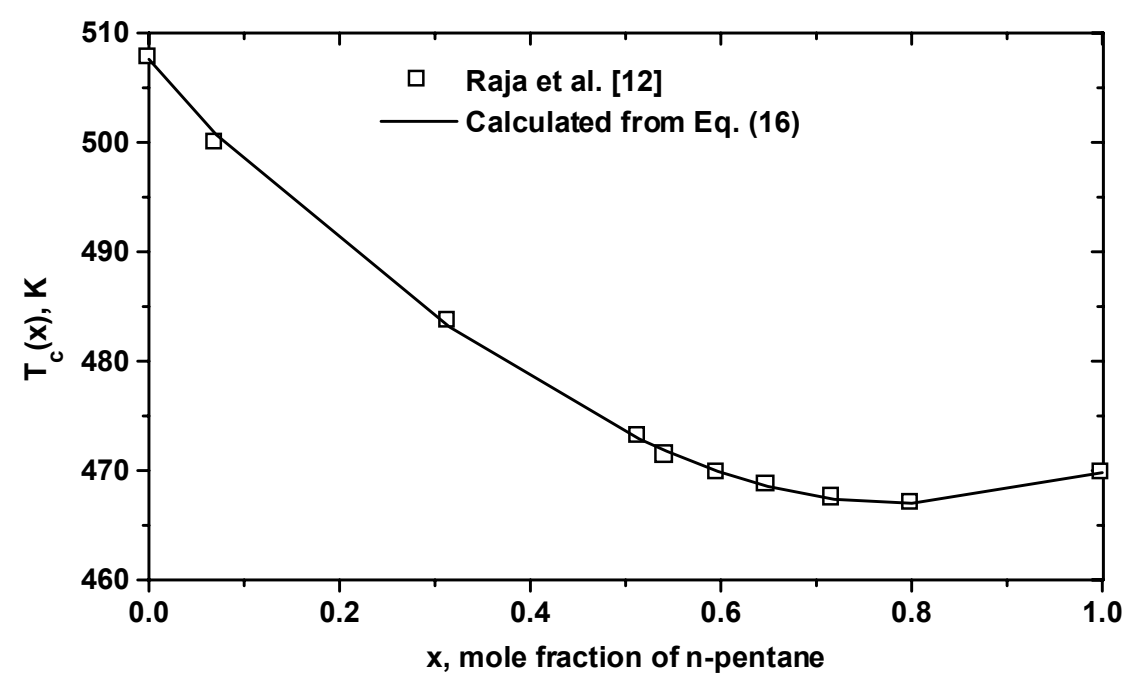

Figure 1: Representation of the critical temperature as a function of the concentration. The curve represents the values calculated from Eq. (16). The squares represent the experimental data obtained by Raja et al. [12].

\section{Conclusion}

For the formulation of any thermodynamic surface for binary mixtures [13-15] we need the analytical relations of the critical parameters as a function of the concentration and the reason we present this work is to make researcher aware of the critical point azeotropy misunderstanding. Therefore, whenever there is a need to formulate a descriptive global equation of state for azeotropic binary mixtures we need to give a special attention of this special point.

\section{Acknowledgments:}

Part of this work is taken from the unpublished work drawn from the author's Ph.D. dissertation [2]. The author is indebted to the late H. R. van den Berg for his assistance in the analysis of the critical line data. Part of this research was done at the University of Maryland. We acknowledge the financial support from the Agence Nationale pour le développement de la Recherche Universitaire (ANDRU) for the financial support under grant No CU 39718. 


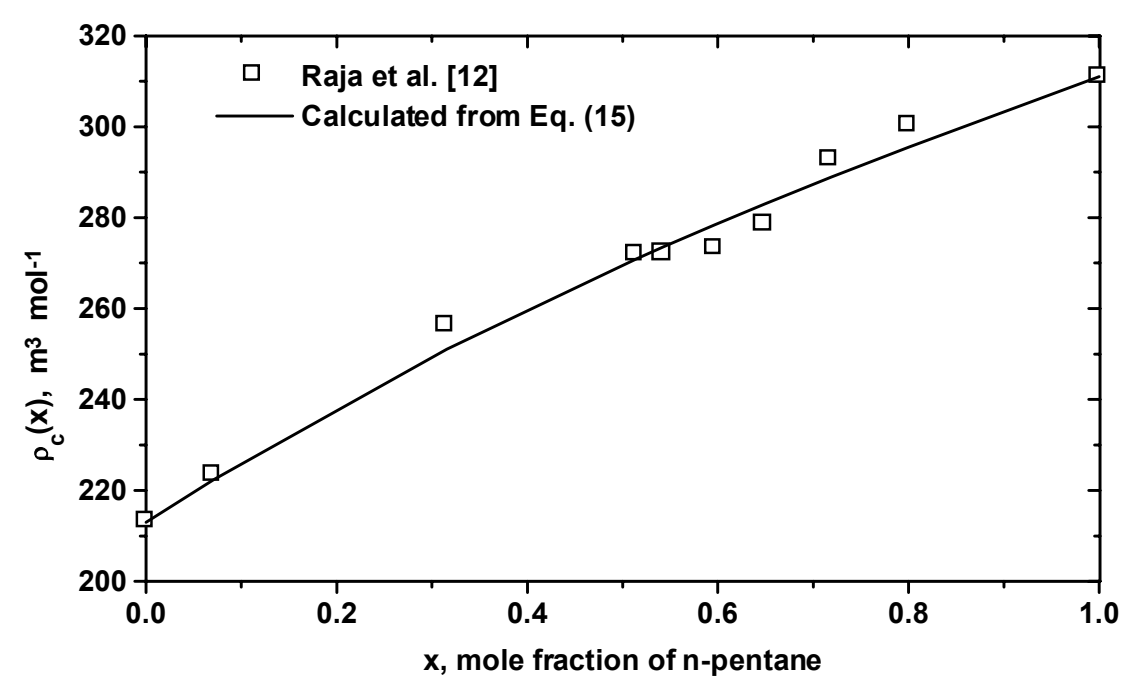

Figure 2: Representation of the critical density as a function of the concentration. The curve represents the values calculated from Eq. (15). The squares represent the experimental data obtained by Raja et al. [12].

\section{References and notes}

1. van Konynenburg, J. P., and Scot, R. L. Critical Lines and Phase Equilibria in Binary van der Waals Mixtures. Phil. Trans. Roy. Soc., London, 1980, 298, 495-540.

2. Abbaci, A. Global Thermodynamic Behavior of Fluids and Fluid Mixtures in the Critical Region. Ph.D. Thesis 1991, University of Maryland at College Park.

3. Rowlinson, J. S., and Swinton, F. L. Liquid and Liquid Mixtures, Butterworth, London, 1982.

4. Griffiths, R. B., and Wheeler, J. C. Critical Points in Multi-Component Systems. Phys. Rev. A 1970, 2, 1047-1064.

5. Leung, S. S., and Griffiths, R. B. Thermodynamic Properties near the Liquid-Vapor Critical Line in Mixtures of $\mathrm{He}^{3}$ and $\mathrm{He}^{4}$. Phys. Rev. A 1973, 8, 2670-2683.

6. Moldover, M. R., and Gallagher, J. S. Critical Points of Mixtures: Analogy with Pure Fluids. AIChE J. 1978, 24, 267-278.

7. Levelt Sengers, J. M. H., Greer, W. L., and Sengers, J. V. Scaled Equation of State Parameters for Gases in the Critical Region. J. Phys. Chem. Ref. Data 1976, 5, 1-49.

8. Khazanova, N. E., and Lesnevskaya, L. S. Phase and Volume Relations in the System EthaneCarbon Dioxide. Russ. J. Phys. Chem. 1967, 41(9), 1279-1282.

9. Khazanova, N. E., and Sominskaya, E. E. Abrupt Changes in Certain Derivatives at the Critical Point of the Azeotropic Mixture. Russ. J. Phys. Chem. 1968, 42(2), 300-301. 
10. Khazanova, N. E., Lesnevskaya, L. S., and Rovoskii, M. B. Azeotropic Systems at High Pressures. Russ. J. Phys. Chem. 1978, 52(4), 521-524.

11. Abbaci, A., van den Berg, H. R., Sakonidou, E., and Sengers J. V. Critical Parameters of Mixtures of Carbon Dioxide and Ethane. Int. J. Thermophys. 1992, 13, 1043-1052.

12. Raja, F. H, Cherry, R. H., and Kay, W. B. Critical Properties of the Vapor-Liquid Equilibria of the Binary System Acetone-n-Pentane. Fluid Phase Equilibria 1986, 25, 137-146.

13. Mattedi, S., Tavares, F. W., and Castier M. Calculation of Mixtures Critical Diagrams Using an Equation of State Based on the Lattice Fluid Theory. Braz. J. Chem. Eng. 2000 N$^{\circ}$ 4-7, 17, 771-784.

14. Anisimov, M. A., and Sengers, J. V. Equations of State for Fluids and Fluid Mixtures. IUPAC, Elsevier, eds.; Sengers, J. V., Kaiser R. F, and Peters C. J. and White J. R., 2000, 381-434.

15. Cheng, H., Anisimov, M. A., and Sengers, J. V. Prediction of Thermodynamic and Transport Properties in the One-Phase Region of Methane-n-Hexane Mixtures Near the Critical End Points. Physica 1997, 128, 67-96.

(C) 2003 by MDPI (http://www.mdpi.org). Reproduction for noncommercial purposes permitted. 